\section{Detecção do risco para internação hospitalar em população idosa: um estudo a partir da porta de entrada no sistema de saúde suplementar}

\author{
Screening for risk of hospitalization in the elderly: \\ a study based on a single entry point in a health \\ maintenance organization in Brazil
}

\author{
${ }_{1}^{1}$ Dix-Amico Saúde, Rio de \\ Janeiro, Brasil. \\ 2 Instituto de Medicina \\ Social, Universidade do \\ Estado do Rio de Janeiro, \\ Rio de Janeiro, Brasil. \\ ${ }^{3}$ Hospital Estadual Getúlio \\ Vargas, Rio de Janeiro, Brasil. \\ ${ }^{4}$ Faculdade de Enfermagem, \\ Universidade do Estado do \\ Rio de Janeiro, Rio de Janeiro, \\ Brasil \\ Correspondência \\ K. Estrella \\ Dix-Amico Saúde. \\ Rua Tenente Possolo 33, \\ Rio de Janeiro, $R J$ \\ 20230-160, Brasil. \\ estrella@webdigital.com.br
}

\begin{abstract}
Population aging requires new strategies for the evaluation and care of different elderly groups. The aim of this study was to present a single entry point model for health care to the elderly in a health maintenance organization. The model prioritizes care for individuals at greatest risk, defining the groups followed subsequently under a specific program for prevention and care. Telephone interviews were used to evaluate the probability of hospitalization for 2,637 users of the health plan. 53.9\% of the individuals who were contacted agreed to participate in the sur$v e y$, and an index was calculated to estimate the probability of hospitalization. 3.23\% of subjects were classified as high risk, $7.23 \%$ as mediumhigh risk, and $13.4 \%$ as medium risk. Subsequent prioritization of care was based on this stratification. The model will enable improved planning and definition of priorities for resource allocation within a health care system subject to serious funding limitations. Studies that identify criteria for the distribution of health care resources are thus essential to the success of consistent and realistic health policies for the elderly.
\end{abstract}

Aged; Hospitalization; Supplemental Health
Kylza Estrella 1,2

Claudia E. F. Oliveira 1

Anne A. Sant'Anna 1,3

Célia Pereira Caldas 4

\section{Introdução}

O presente trabalho apresenta proposta de hierarquização da demanda de idosos em sistema de saúde complementar, baseado na identificação dos indivíduos com maior risco para internação hospitalar.

Essa preocupação é justificada pela constatação de que a população idosa apresenta alta utilização dos serviços de saúde e elevado índice de permanência e reinternação hospitalar ${ }^{1}$. Esses fatos são mais alarmantes quando analisados dentro do contexto do progressivo envelhecimento populacional e do crescente custo médico conjugado ao avanço da tecnologia em saúde 2 .

Semelhante cenário demanda a identificação da população idosa com maior risco de adoecimento para otimizar a adoção de medidas que organizem intervenções assistenciais e preventivas, garantindo que a tomada de decisão em relação à escolha de prioridades seja feita de forma equânime e eficiente.

O modelo estudado parte da aplicação de um questionário que busca identificar os sujeitos em risco de internação.

Portanto, este estudo tem como propósito apresentar uma hierarquização da demanda de idosos em sistema de saúde complementar de uma operadora de saúde na cidade do Rio de Janeiro, Brasil, através da aplicação de um questionário, a fim de subsidiar tomada de decisões para a alocação de recursos. 


\section{Métodos}

Foi realizado um estudo transversal com todos os idosos ( $\geq 65$ anos) usuários da carteira de pessoa física de uma operadora de saúde da cidade do Rio de Janeiro, de outubro a novembro de 2005. Por ocasião da coleta de dados havia 2.637 idosos nesta carteira. Todos foram incluídos no estudo. As respostas foram obtidas do próprio idoso e, quando era impossível que o próprio o fizesse, do seu cuidador. Foram definidos como procedimentos: utilização de instrumento probabilty of repetead admission (PRA) 3; aplicação do instrumento por meio de telefone; e análise das respostas por meio de estatísticos, a fim de identificar perfis de risco.

O instrumento PRA foi desenvolvido por Boult et al. 3, a partir de um estudo de coorte. Inicialmente, o instrumento relacionou 28 variáveis com hospitalização e mortalidade subseqüentes. Foi desenvolvido um modelo de regressão logística - técnica usada para a análise de dados com resposta binária (no caso, risco ou não de internação) - para o exame das variáveis de maior poder preditor, definindo, ao final, o instrumento para a avaliação da probabilidade de admissão hospitalar, denominado probability of repeated admission, (probabilidade de internação repetida), abreviado PRA ${ }^{3}$.

Boult et al. 3 verificaram os seguintes fatores de risco como sendo indicadores com maior poder de predição para admissão hospitalar: auto-percepção do estado de saúde, número de pernoite hospitalar e número de visitas médicas nos últimos 12 meses, presença de diabetes mellitus, presença de doença cardíaca, sexo, presença de cuidador e faixa etária. A partir desses fatores, foi construído um questionário de oito perguntas, que, através da aplicação de regressão logística ${ }^{4}$, define um coeficiente que indica a probabilidade de internação repetida. Os valores possíveis para o coeficiente PRA vão de 0,07-0,78, sendo que os autores propõem um limite de 0,5 na definição de grupos de alto e baixo risco para internação. E, para encaminhamento de idosos para avaliação e reabilitação, utilizaram o ponto de corte de 0,45 . No serviço em estudado foi adotado o ponto de corte de 0,30 , para captação de indivíduos para o programa. A decisão de adotar essa classificação de risco deu-se fundamentalmente pela intenção de expandir a cobertura do programa de prevenção para um número maior de usuários. É relevante a compreensão de que, no ambiente da medicina suplementar em questão, não há qualquer serviço que considere a especificidade da população idosa.
Vale ressaltar que este instrumento foi validado para triagem e estratificação de idosos de risco 5,6, já tendo sido inclusive avaliado em nosso meio ${ }^{1}$.

Devido à ausência, em nosso meio, de referência à utilização de questionário de saúde através do correio, o que nos permite supor um baixo índice de retorno, e considerando a ampla presença do serviço de telefonia fixa entre a população urbana a que se destina a consulta, optamos pela aplicação do instrumento PRA por telefone.

Na primeira fase do trabalho, foi contratada uma empresa de call center ativo para a aplicação do questionário, através de contato telefônico a ser efetuado por dois operadores previamente treinados. A atividade realizou-se com um programa que possibilita o controle desses contatos, isto é, define o número total realizado, quantos por indivíduo da população-alvo e o seu desfecho. A aplicação transcorreu em regime de 12 horas diurnas, durante um mês, de segunda a sábado. Essa fase foi monitorada durante a primeira semana por enfermeira da equipe de saúde da operadora, ouvindo o diálogo idoso-operador para correção de possíveis equívocos por parte dos operadores. Após esse período, a enfermeira passou a atuar realizando a supervisão semanal do trabalho de campo.

Cada tentativa fracassada de contato telefônico era repetida até dez vezes pelo operador em horários diferentes. O sistema de informática da operadora de call center define, automaticamente, um mecanismo de fila e controle da mesma, para contato com a população-alvo.

O questionário era apresentado pelo operador como parte de uma avaliação dos indivíduos idosos da operadora de saúde, dentro de uma visão de prevenção de saúde do programa de idosos. Relatórios foram enviados para o programa na operadora de saúde semanalmente para acompanhamento do desenvolvimento da aplicação da pesquisa.

Ao final da fase de aplicação do questionário, os dados foram importados para novo arquivo para realização do cálculo do coeficiente de risco de cada idoso segundo aplicação de regressão logística. Esses resultados foram tratados estatisticamente para serem apresentados em percentuais.

O risco foi considerado baixo quando o PRA apresentava-se menor do que 0,30; médio de 0,30-0,39; médio-alto de 0,400-0,499 e alto, maior ou igual a 0,500. A decisão de adotar essa classificação de risco deu-se fundamentalmente pela intenção de expandir a cobertura do programa de prevenção para um número maior de usuários e a compreensão que, no ambiente 
da medicina suplementar em questão, não há serviços que considerem a especificidade da população idosa. Dessa maneira, a captação para o programa se dá, de acordo com uma escala decrescente de valores para PRA, de forma a convidar inicialmente os indivíduos com maior probabilidade de internação hospitalar.

\section{Resultados}

Dos 2.637 idosos da população-alvo, 1.423 (53,9\%) responderam ao PRA e $1.214(46,1 \%)$ não responderam. Entre os que não responderam, não foi conseguido contato telefônico com 1.073 pessoas e 141 pessoas se recusaram a participar.

Dos 1.423 idosos estudados, 1.334 (93,7\%) contavam com uma estrutura de apoio. Quanto à faixa etária, $760(53,4 \%)$ apresentavam entre 65 a 74 anos, $335(9,5 \%)$ entre 75 a 79 anos, $193(13,6 \%)$ entre 80 a 84 anos e $135(23,5 \%)$ mais de 85 anos, sendo $1.023(71,9 \%)$ mulheres e 400 $(28,2 \%)$ homens.

Quanto à distribuição dos fatores de risco na população estudada (Tabela 1), a auto-percepção do estado de saúde mostrou que 163 (11,4\%) pessoas consideram sua saúde excelente; 186 $(13,1 \%)$ muito boa; $688(48,3 \%)$ boa; 348 (24,5\%) média; e 38 (2,7\%) ruim.

Nos últimos 12 meses anteriores à entrevista 1.217 (85,5\%) pessoas não haviam passado por internação hospitalar; 173 (12,2\%) pessoas passaram por uma internação; $22(1,5 \%)$ passaram por duas a três internações e $11(0,8 \%)$ pessoas foram internadas mais de três vezes.

Quanto ao número de consultas médicas nos últimos 12 meses anteriores à entrevista, foi verificado que $188(13,2 \%)$ pessoas não se consultaram; $256(17,9 \%)$ se consultaram apenas uma vez; $510(35,8 \%)$ pessoas se consultaram 2 a 3 vezes; 307 (21,6\%) se consultaram 4 a 6 vezes e $162(11,4 \%)$ se consultaram mais de 6 vezes.

Em relação à presença de diagnósticos clínicos de condições crônicas consideradas de risco para internação, 170 (11,9\%) pessoas apresentavam diabetes mellitus e 154 (10,8\%) apresentavam doença cardíaca (angina, infarto miocárdio, doença coronariana), nos últimos 12 meses anteriores à entrevista.

A Tabela 1 resume os perfis encontrados na população investigada, com relação aos fatores de risco de hospitalização, conforme propõe o instrumento PRA (Figura 1).

Perfis das respostas obtidas sobre fatores de risco na população estudada.

\begin{tabular}{|c|c|c|}
\hline & $\mathbf{n}$ & $\%$ \\
\hline \multicolumn{3}{|c|}{ 1. Auto-percepção do estado saúde } \\
\hline Excelente & 163 & 11,4 \\
\hline Muito boa & 186 & 13,1 \\
\hline Boa & 688 & 48,3 \\
\hline Média & 348 & 24,5 \\
\hline Ruim & 38 & 2,7 \\
\hline \multicolumn{3}{|c|}{ 2. Número de internações nos últimos 12 meses anteriores à entrevista } \\
\hline Nenhuma vez & 1.217 & 85,5 \\
\hline Uma vez & 173 & 12,2 \\
\hline Duas a três vezes & 22 & 1,5 \\
\hline Mais do que três vezes & 11 & 0,8 \\
\hline \multicolumn{3}{|c|}{ 3. Número de consultas médicas nos últimos 12 meses anteriores à entrevista } \\
\hline Nenhuma & 188 & 13,2 \\
\hline Uma vez & 256 & 17,9 \\
\hline Duas a três vezes & 510 & 35,8 \\
\hline Quatro a seis vezes & 307 & 21,6 \\
\hline Mais do que seis vezes & 162 & 11,4 \\
\hline \multicolumn{3}{|c|}{ 4. Presença de diabetes mellitus nos últimos 12 meses anteriores à entrevista } \\
\hline $\operatorname{Sim}$ & 170 & 11,9 \\
\hline \multicolumn{3}{|c|}{ 5. Presença de doença cardíaca (angina, infarto miocárdio, doença coronariana) } \\
\hline Sim & 154 & 10,8 \\
\hline
\end{tabular}


Figura 1

Instrumento de avaliação de risco de internação hospitalar.

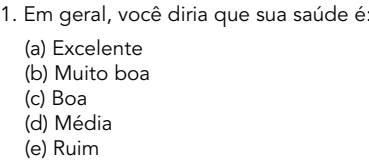

2. Nos últimos 12 meses, você passou a noite como paciente em algum hospital? (a) Não, nenhuma vez (b) Uma vez

(c) Duas ou três vezes

(d) Duas ou três veze

(e) Mais do que três vezes

3. Nos últimos 12 meses, quantas vezes você procurou o médico ou uma clínica para tratamento? (a) Nenhuma vez

(b) Uma vez

(c) Duas ou três vezes

(d) Quatro a seis vezes

(e) Mais do que seis vezes

4. Nos últimos 12 meses, você teve diabetes?

(a) $\mathrm{Sim}$

(b) Não

5. Você alguma vez já teve doença coronariana? Angina? Infarto do miocárdio? Ataque do coração? (a) Sim (qualquer das respostas acima for "sim")

(b) Não (se todas as respostas às questões acima forem "não")

6. Sexo?

(a) Homem

(b) Mulher

7. Existe algum amigo, parente ou vizinho que poderia tomar conta de você por alguns dias se você precisar?

(a) $\mathrm{Sim}$

(b) Não

8. Qual sua data de nascimento? (idade deve então ser calculada)

(a) $65-74$ anos

(b) $75-79$ anos

(c) $80-84$ anos

(d) Mais que 85 anos alto; e $46(3,23 \%)$ pessoas apresentavam risco alto. A distribuição das variáveis conforme o instrumento PRA encontra-se resumida na Tabela 2, apresentada a seguir.

\section{Discussão}

O presente trabalho apresenta uma proposta de avaliação e estratificação de uma população idosa em um serviço de saúde no contexto da medicina suplementar, tendo em vista a necessidade de priorizar o atendimento de indivíduos com maior risco de adoecer. O envelhecimento populacional nos obriga a definir estratégias adequadas para cada grupo de risco nessa população, com medidas de assistência, prevenção e promoção de saúde específicas. Para isso, se faz necessário organizar triagens para estratificação da população, para a orientação de porta de entrada para programas específicos, visando a otimização da utilização dos serviços e maior benefício para os usuários.

O modelo proposto organiza-se de modo complexo, pois parte da identificação da população-alvo, a seguir, utiliza um sistema de informática já existente em operadoras de call center ativo para aplicação de questionário, importa os dados para novo banco que se destina à realização do cálculo do coeficiente propriamente dito. A integração das áreas de saúde e informática é fundamental para viabilizar esse processo.

O sucesso de resposta de $54 \%$ na aplicação do questionário foi considerado satisfatório. Pacala et al. 7 obtiveram retorno do mesmo questionário por correio de $60,5 \%$, inclusive com reforço de novo exemplar do questionário para não respondentes, enviando o questionário pelo correio. É interessante observar que apenas $11,6 \%$ da população se recusou a responder o questionário pelo telefone. O maior impasse em relação ao uso do meio telefônico para aplicação do questionário é definido por problemas relativos à telefonia, aqui representados por telefones que não atendem, ocupados e errados. Nesse aspecto, vale ressaltar a importância da atualização dos bancos de dados dos serviços de saúde. A utilização do recurso telefônico é de baixo custo e rápida, lembrando que em apenas um mês de trabalho foi possível realizar a execução do questionário.

O universo de 2.637 indivíduos dividiu-se inicialmente em duas categorias de não respondentes $(46,1 \%)$ e pesquisados $(53,9 \%)$. No universo pesquisado, encontramos a presença marcante do sexo feminino $(71,9 \%)$. É fato reconhecido a busca pelos serviços de saúde por parte da popu- 
lação feminina 8 , o que parece estar reproduzido aqui, na medida em que mais mulheres respondem ao questionário ou estão disponíveis para participar da pesquisa. De acordo com Goldani ${ }^{9}$, homens e mulheres vivem e envelhecem de forma diferente. Considerada como vantagem e indicador de melhor qualidade de vida, a maior longevidade da mulher acaba sendo, também, um de seus problemas. A maior esperança de vida faz com que muitas das mulheres idosas passem pela experiência de debilitação biológica devido a doenças crônicas, enquanto os homens morrem antes e de causas do tipo acidentes cardiovasculares.

No tocante à idade, a faixa etária predominante é aquela entre 65 a 74 anos, merecendo destaque a faixa etária acima de 80 anos $(37,1 \%)$, devido à conhecida fragilidade neste grupo 10 .

Quase a totalidade dos entrevistados relata ter apoio social $(93,7 \%)$, caso necessite de internação. Esse achado é semelhante a estudo anterior onde também foi elevada a proporção de idosos que contavam com ajuda em caso de necessidade 8 .

A presença de diabetes mellitus e doenças cardíacas tiveram valores aproximados (respectivamente $11,9 \%$ e 10,8\%). Pesquisa anterior, em nosso meio, com o mesmo instrumento em ambiente ambulatorial especializado em idosos do Sistema Único de Saúde (SUS), revelou uma prevalência $22,9 \%$ para diabetes mellitus e $30 \%$ para doenças cardiovasculares 8 .

Em relação ao número de consultas médicas, essa utilização é elevada, uma vez que $33 \%$ da amostra teve quatro ou mais consultas médicas nos últimos 12 meses, considerando que a média no serviço é de três a quatro consultas por ano. Esse número é igualmente elevado quando comparado com a média de 2,79 consultas por habitante/ano no SUS na Região Metropolitana do Rio de Janeiro (Departamento de Informática do SUS; http://www.datasus.gov.br). Por outro lado, em estudo anterior, a freqüência de consultas foi elevada, com $61,9 \%$ da amostra referindo ter tido quatro ou mais consultas nos 12 meses prévios à entrevista ${ }^{8}$. Esse dado aponta para a alta utilização de serviços de saúde da população idosa.

Finalmente, 14,5\% dos entrevistados foram internados ao menos uma vez nos últimos 12 meses anteriores à entrevista, praticamente 0 dobro do encontrado em pesquisa anterior 8 . Esse achado sugere a prevalência de uma população idosa de alto risco no contexto da medicina suplementar, considerando ser hospitalização um preditor importante de mortalidade em nosso meio 11.
Tabela 2

Distribuição dos valores de PRA (probabilty of repetead admission) na população estudada.

\begin{tabular}{lccc}
\hline $\begin{array}{l}\text { Estratificação de risco } \\
\text { de hospitalização }\end{array}$ & PRA & $\mathbf{n}$ & $\%$ \\
\hline Baixo & & & \\
Médio & $<0,30$ & 1.082 & 76,03 \\
Médio-alto & $0,30-0,39$ & 192 & 13,4 \\
Alto & $0,400-0,499$ & 103 & 7,23 \\
& 0,500 & 46 & 3,23 \\
\hline
\end{tabular}

\section{Conclusão}

Ao se calcular o coeficiente de probabilidade de internação hospitalar a partir desses dados, é possível ter um parâmetro para decidir quais os idosos que deverão ter prioridade para receber a intervenção do programa. Por outro lado, é preciso destacar que o PRA significa uma probabilidade e representa uma combinação de fatores. Isso significa que pode ser considerado um indicador para medidas preventivas. Ou seja, mesmo diante daqueles usuários com PRA elevado sem registro de internação, pode-se iniciar um trabalho preventivo, para em um segundo momento, com a intervenção do programa, implementar medidas assistenciais e preventivas para a população com maior risco. Enfim, ao definir a estratificação da população de acordo com o risco, é possível melhorar o planejamento e gestão do próprio serviço, pois passa a ser possível dimensionar o quantitativo de indivíduos para intervenção e planejar a assistência.

O objetivo da proposta estudada é priorizar o atendimentodosgruposdealto, médio-altoemédio risco, em seqüência. É importante observar que $23,8 \%$ do grupo respondente estão inseridos nessas categorias entre médio e alto risco, proporção apontada como sendo a correspondente àquela do idoso frágil na população geral 7,12 . Portanto, é esse grupo que deve ser priorizado no planejamento de ações de saúde.

O grupo de baixo risco precisa ser atendido preventivamente, porém, suas demandas podem ser avaliadas após o atendimento daqueles que se encontram no grupo de risco, em uma nova escala de agendamento de prioridades para a assistência específica. É preciso destacar que no sistema de saúde suplementar, o grupo de baixo risco encontra pleno acesso e escolha de atendimento médico, não sofrendo em qualquer instância, limites à assistência. A questão que se impõe é a necessidade de definir prioridades dentro de um universo restrito em recursos de saúde. 


\section{Resumo}

O envelhecimento populacional impõe a necessidade de criar estratégias de avaliação e acompanhamento para os diferentes grupos da população idosa. O objetivo do artigo é apresentar uma proposta de hierarquização da demanda de idosos em sistema de saúde complementar, com base no uso de instrumento de detecção do risco de internação hospitalar. Esse instrumento foi aplicado por meio telefônico em 2.637 usuários do plano de saúde. A seguir, realizou-se o cálculo do coeficiente de risco de cada idoso, segundo aplicação de regressão logística. A partir dessas respostas calculou-se o indice de probabilidade de admissão hospitalar: 3,23\% encontravam-se nos grupos de alto risco; 7,23\% de médio-alto, e 13,4\% apresentaram médio risco. O modelo permitiu a hierarquização da demanda da população de idosos, de acordo com o risco de internação hospitalar, o que proporcionou melhorar o planejamento e gestão do serviço, pois passou a ser possivel dimensionar o quantitativo de indivíduos para intervenção e planejar a assistência. Portanto, estudos que apontem critérios para a distribuição de recursos são cada vez mais imprescindiveis para sustentar políticas de gestão realistas e consistentes.

Idoso; Hospitalização; Saúde Suplementar

\section{Referências}

1. Veras RP, Lourenço R, Martins C, Sanchez MA, Chaves PH. Novos paradigmas do modelo assistencial no setor saúde: conseqüência da explosão populacional dos idosos no Brasil. In: Veras RP, organizador. Terceira idade: gestão contemporânea em saúde. Rio de Janeiro: Editora Relume Dumará/Universidade Aberta da Terceira Idade, Universidade do Estado do Rio de Janeiro; 2002. p. 11-79.

2. Boult C, Pacala J. Care of older people at risk. In: Calkins E, editor. New ways to care for older people: building systems based on evidence. New York: Springer Publishing Company; 1999. p. 65-84.

3. Boult C, Dowd B, McCaffrey D, Boult L, Hernandez R, Krulewitch H. Screening elder for risk of hospital admission. J Am Geriatr Soc 1993; 41:811-7.

4. Kleinbaum D. Logistic regression a self-learning text. New York: Springer Publishing Company; 1994.

5. Boult L, Boult C, Pirie P, Pacala JT. Test-retest reliability of a questionnaire that identifies elders at risk for hospital admission. J Am Geriatr Soc 1994; 42:707-11.

6. Pacala JT, Boult C, Boult L. Predictive validity of the Pra instrument among older recipients of managed care. J Am Geriatr Soc 1997; 45:614-7.

7. Pacala JT, Boult C, Boult L. Predictive validity of a questionnaire that identifies older persons at risk for hospital admission. J Am Geriatr Soc 1995; 43:374-7.

\section{Colaboradores}

K. Estrella participou do planejamento da pesquisa, análise de dados, elaboração e redação do artigo. A. A. Sant'Anna redigiu o artigo. C. E. F. Oliveira foi responsável direta pela coleta de dados, redação do manuscrito do artigo e análise da versão final para publicação. C. P. Caldas contribuiu na supervisão e análise crítica do artigo.
8. Veras RP, organizador. Terceira idade: gestão contemporânea em saúde. Rio de Janeiro: Editora Relume Dumará/Universidade Aberta da Terceira Idade, Universidade do Estado do Rio de Janeiro; 2002.

9. Goldani AM. Mulheres e envelhecimento: desafios para novos contratos intergeracionais e de gênero. In: Camarano AA, organizador. Muito além dos 60: os novos idosos brasileiros. Rio de Janeiro: Instituto de Pesquisa Econômica Aplicada; 1999. p. 75-114.

10. Siqueira AB, Cordeiro RC, Perracini MR, Ramos LR. Impacto funcional da internação hospitalar de pacientes idosos. Rev Saúde Pública 2004; 38:687-94.

11. Ramos LR, Simões EJ, Albert MS. Dependence in activities of daily living and cognitive impairment strongly predicted mortality in older urban residents in Brazil: a 2-year follow up. J Am Geriatr Soc 2001; 49:1168-75.

12. Veras R. País jovem com cabelos brancos: a saúde do idoso no Brasil. Rio de Janeiro: Editora RelumeDumará; 1994.

Recebido em 19/Out/2007

Versão final reapresentada em 14/Mai/2008

Aprovado em 26/Mai/2008 